\title{
AN INFINITE CLASS OF PERIODIC SOLUTKONS OF PERIODICALLY PERTURBED DUFFING EQUATIONS AT RESONANCE
}

\author{
TUNG-REN DING
}

\begin{abstract}
In this paper, by using a generalized form of the PoincaréBirkhoff Theorem, we demonstrate that the Duffing equation

$$
\frac{d^{2} x}{d t^{2}}+g(x)=p(t) \quad(\equiv p(t+2 \pi))
$$

may also admit an infinite number of $2 \pi$-periodic solutions even in a resonance case.
\end{abstract}

1. In this paper we study the existence problem of periodic solutions for the Duffing equation

$$
\frac{d^{2} x}{d t^{2}}+g(x)=p(t)
$$

where $g(x), p(t) \in C(R, R)$ and $p(t)$ is $2 \pi$-periodic.

In the super-linear case, that is,

$$
\lim _{|x| \rightarrow \infty}\left[x^{-1} g(x)\right]=\infty,
$$

it has been proved in a recent paper by W. Y. Ding that (1.1) has infinitely many $2 \pi$-periodic solutions [1].

D. E. Leach proved the existence and uniqueness of $2 \pi$-periodic solution of (1.1) under the following Loud condition

$$
m^{2}<\lambda \leq g^{\prime}(x) \leq \mu<(m+1)^{2} \quad(g(0)=0),
$$

with a given integer $m \geq 0$ and two such constants $\lambda$ and $\mu$ [2]. R. Reissig proved the existence of periodic solutions of (1.1) under a weaker condition [3]

$$
m^{2}<\lambda \leq x^{-1} g(x) \leq \mu<(m+1)^{2}, \quad|x| \geq a>0 .
$$

The crucial point of the conditions above is to exclude the resonance cases. At resonance, (1.1) may have no bounded solution [6]; therefore, the existence problem of periodic solutions challenges attention. A. C. Lazer and D. E. Leach, and L. Césari succeeded to prove the existence of $2 \pi$-periodic solutions of (1.1) at a point of resonance provided that $h(x)=g(x)-m^{2} x$ is bounded and some other additional conditions are assumed $[4,5]$. In a recent paper, the present author resolved the existence problem of $2 \pi$-periodic solutions of (1.1) under a weakened version of (1.2) [6]:

$$
m^{2} \leq g^{\prime}(x) \leq(m+1)^{2} \quad(g(0)=0) .
$$

Received by the editors February 25, 1981.

1980 Mathematics Subject Classification. Primary 34C25; Secondary 70K20.

Key words and phrases. Duffing equation, resonance, periodic solutions, a generalized PoincaréBirkhoff Theorem. 
In this paper, by using a generalized form of the Poincaré-Birkhoff Theorem due to W. Y. Ding, we will demonstrate that (1.1) may also admit an infinite number of $2 \pi$-periodic solutions even in a resonance case. This shows once more the complexity of (1.1) at resonance.

2. We need the following hypotheses:

$\left(\mathrm{H}_{1}\right)$ Let $g(x) \in C^{\prime}(R, R)$, and let $K$ be a positive constant, such that

$$
\left|g^{\prime}(x)\right| \leq K, \quad x \in R
$$

$\left(\mathrm{H}_{2}\right)$ There exist two constants $A_{0}>0$ and $M_{0}>0$, such that

$$
x^{-1} g(x) \geq A_{0}, \quad|x| \geq M_{0} .
$$

Then we consider the auxiliary equation

$$
\frac{d^{2} z}{d t^{2}}+g(z)=0
$$

and its equivalent system

$$
\frac{d z}{d t}=w, \quad \frac{d w}{d t}=-g(z)
$$

This is a planar autonomous system whose orbits are curves determined by the following equation

$$
V(z, w) \equiv \frac{1}{2} w^{2}+G(z)=c,
$$

where $G(z)=\int_{0}^{z} g(u) d u$ and $c$ is a parameter.

The hypothesis $\left(\mathrm{H}_{2}\right)$ obviously implies

$$
\lim _{|z| \rightarrow \infty} G(z)=\infty \text { and } \lim _{|z|+|w| \rightarrow \infty} V(z, w)=\infty
$$

It follows that the set $V^{-1}(c)$ is compact. Furthermore, $\left(\mathrm{H}_{2}\right)$ yields that there exist constants $C_{0}>0$ and $A_{1}>0$ such that if $c \geq c_{0}$, then

$$
w^{2}+z g(z) \geq A_{1}\left(z^{2}+w^{2}\right), \quad(z, w) \in V^{-1}(c) .
$$

Note that the left-hand member of the last inequality is just the directional derivative of $V(z, w)$ along the vector $(z, w)$. Therefore, there is no critical point in $V^{-1}(c)$ for $c \geq c_{0}$, and $V^{-1}(c)$ is a compact one-dimensional manifold. Furthermore $V^{-1}(c)$ is star-shaped about the origin. We have thus proved

LEMMA 2.1. If $\left(\mathrm{H}_{2}\right)$ holds, then $V^{-1}(c)$ is a closed curve for $c \geq c_{0}$ which is star-shaped about the origin 0.

In the sequel, we will denote the curve $V^{-1}(c)$ by $\Gamma_{c}$. It follows from Lemma 2.1 that each curve $\Gamma_{c}\left(c \geq c_{0}\right)$ intersects the $z$-axis at two points: $(h(c), 0)$ and $\left(-h_{1}(c), 0\right)$, where $h(c)>0$ and $h_{1}(c)>0$ are uniquely determined by the formula

$$
G(h(c))=G\left(-h_{1}(c)\right)=c .
$$

Let $(z(t), w(t))$ be any solution of $\left(2.1^{\prime}\right)$ whose orbit is $\Gamma_{c}\left(c \geq c_{0}\right)$. Clearly, this solution is periodic. Let $\tau(c)$ denote the least positive period of this solution. It follows from the equation (2.2) that

$$
\tau(c)=\sqrt{2} \int_{-h_{1}(c)}^{h(c)} \frac{d u}{\sqrt{c-G(u)}} .
$$


To obtain the desired result, we need another hypothesis:

$\left(\mathrm{H}_{3}\right)$ There exist a constant $\alpha>0$, an integer $m>0$, and two sequences $\left\{a_{k}\right\}$ and $\left\{b_{k}\right\}$, such that $a_{k} \rightarrow \infty$ and $b_{k} \rightarrow \infty$ as $k \rightarrow \infty$; and moreover

$$
\tau\left(a_{k}\right)<\frac{2 \pi}{m}-\alpha, \quad \tau\left(b_{k}\right)>\frac{2 \pi}{m}+\alpha .
$$

Now we are in a position to state the main result of this paper.

THEOREM 2.1. Assume $\left(\mathrm{H}_{1}\right)-\left(\mathrm{H}_{3}\right)$ hold. Then equation (1.1) has infinitely many $2 \pi$-periodic solutions.

A concrete example for applications of this theorem will be given in $\S 5$.

3. To prove Theorem 2.1, we briefly restate a generalized form of the PoincaréBirkhoff fixed point theorem in Theorem A below [7].

Let $D$ denote an annular region in the $(x, y)$-plane. The boundary of $D$ consists of two simple closed curves: the inner boundary curve $\mathbf{C}_{1}$ and the outer boundary curve $\mathbf{C}_{2}$. Let $D_{1}$ denote the simple connected open set bounded by $\mathbf{C}_{1}$. Consider an area-preserving mapping $T: R^{2} \rightarrow R^{2}$. Suppose that $T(D) \subset R^{2}-\{0\}$, where 0 is the origin. Let $(\gamma, \theta)$ be the polar coordinate of $(x, y)$, that is, $x=\gamma \cos \theta$, $y=\gamma \sin \theta$. Assume the restriction $T \mid D$ is given by

$$
\gamma^{*}=f(\gamma, \theta), \quad \theta^{*}=\theta+g(\gamma, \theta),
$$

where $f$ and $g$ are continuous in $(\gamma, \theta)$, and $2 \pi$-periodic in $\theta$.

THEOREM A. Besides the above-mentioned assumptions, we assume that

(i) $\mathbf{C}_{1}$ is star-shaped about the origin;

(ii) $0 \in T\left(D_{1}\right)$;

(iii) $g(\gamma, \theta)>0(<0),(\gamma \cos \theta, \gamma \sin \theta) \in \mathbf{C}_{1}$; $g(\gamma, \theta)<0(>0),(\gamma \cos \theta, \gamma \sin \theta) \in \mathbf{C}_{2}$.

Then $T$ has at least two fixed points in $D$.

Now, let $\Gamma_{a_{k}}$ and $\Gamma_{b_{k}}$ be the curves given by Lemma 2.1, where the specified parameters $a_{k}, b_{k} \geq c_{0}$ are given by $\left(\mathrm{H}_{3}\right)$, for $k \geq n_{0}$. We may rearrange $\left\{a_{k}\right\}$ and $\left\{b_{k}\right\}$, if necessary, so that $a_{k}<b_{k}<a_{k+1}$ for $k \geq n_{0}$. Then, $\Gamma_{a_{k}}$ and $\Gamma_{b_{k}}$ bound an annular region $A_{k}$, and $\Gamma_{b_{k}}$ and $\Gamma_{a_{k+1}}$ bound another annular region $B_{k}$, for $k \geq n_{0}$.

Let $T: R^{2} \rightarrow R^{2}$ be the Poincaré mapping induced by equation (1.1). It is well known that each fixed point of $T$ corresponds to a $2 \pi$-periodic solution of (1.1). In the following section, we will apply Theorem A to show that $T$ has at least two fixed points in each $A_{k}$ and $B_{k}$ for sufficiently large $k$. As a consequence, (1.1) has an infinite class of $2 \pi$-periodic solutions.

4. Consider the equivalent system of (1.1),

$$
\frac{d x}{d t}=y, \quad \frac{d y}{d t}=-g(x)+p(t)
$$

Let $(\bar{x}(t, x, y), \bar{y}(t, x, y))$ be the solution of (4.1) through the initial point $(\bar{x}(0), \bar{y}(0))=$ $(x, y)$. It is not hard to show that every such solution exists on the whole $t$-axis under condition $\left(\mathrm{H}_{1}\right)$. Then the Poincaré map $T: R^{2} \rightarrow R^{2}$ is well defined by

$$
(x, y) \mapsto(\bar{x}(2 \pi, x, y), \bar{y}(2 \pi, x, y))
$$


It is well known that $T$ is an area-preserving homeomorphism.

By applying the transform $x(t)=\gamma(t) \cos \theta(t), y(t)=\gamma(t) \sin \theta(t)$ to (4.1), we get the equations for $\gamma(t)$ and $\theta(t)$,

$$
\left\{\begin{array}{l}
\frac{d \gamma}{d t}=\gamma \cos \theta \cdot \sin \theta-g(\gamma \cos \theta) \sin \theta+p(t) \sin \theta \\
\frac{d \theta}{d t}=-\sin ^{2} \theta-\frac{1}{\gamma}(g(\gamma \cos \theta) \cos \theta-p(t) \cos \theta)
\end{array}\right.
$$

whenever $\boldsymbol{\gamma} \neq 0$.

Let $(\bar{\gamma}(t, \gamma, \theta), \bar{\theta}(t, \gamma, \theta))$ be the solution of (4.2) through the initial point $(\bar{\gamma}(0), \bar{\theta}(0))$ $=(\gamma, \theta)$. Then the map $T$ can also be written in the polar coordinate form

$$
\gamma^{*}=\bar{\gamma}(2 \pi, \gamma, \theta), \quad \theta^{*}=\bar{\theta}(2 \pi, \gamma, \theta)+2 l \pi,
$$

where $l$ is an arbitrary integer. It can be easily seen that if $(\gamma, \theta)$ is such that

$$
\bar{\gamma}(t, \gamma, \theta)>0, \quad t \in[0,2 \pi],
$$

then $\bar{\theta}(2 \pi, \gamma, \theta)$ is well defined and continuous in $(\gamma, \theta)$, and moreover,

$$
\bar{\theta}(2 \pi, \gamma, \theta+2 \pi)=\bar{\theta}(2 \pi, \gamma, \theta)+2 \pi .
$$

Next, we take the transform $z(t)=\rho(t) \cos \varphi(t), w(t)=\rho(t) \sin \varphi(t)$ for system $\left(2.1^{\prime}\right)$. Then the resulting equations for $\rho(t)$ and $\varphi(t)$ are

$$
\left\{\begin{array}{l}
\frac{d \rho}{d t}=\rho \cos \varphi \cdot \sin \varphi-g(\rho \cos \varphi) \sin \varphi \\
\frac{d \varphi}{d t}=-\sin ^{2} \varphi-\frac{1}{\rho} g(\rho \cos \varphi) \cos \varphi
\end{array}\right.
$$

Let $(\bar{\rho}(t, \rho, \varphi), \bar{\varphi}(t, \rho, \varphi))$ be the solution of (4.6) through the initial point $(\bar{\rho}(0), \bar{\varphi}(0))$ $=(\rho, \varphi)$.

LEMMA 4.1. Let $\Phi(\rho, \varphi)=\bar{\varphi}(2 \pi, \rho, \varphi)-\varphi$, and let $\beta=\min \left\{2 \pi, m \alpha A_{1}\right\}$, where $m, \alpha$ and $A_{1}$ are given in $\left(\mathrm{H}_{3}\right)$ and (2.3). Then we have

$$
\left\{\begin{array}{l}
\Phi(\rho, \varphi) \leq-2 m \pi-\beta, \quad(\rho \cos \varphi, \rho \sin \varphi) \in \Gamma_{a_{k}} \\
\Phi(\rho, \varphi) \geq-2 m \pi+\beta, \quad(\rho \cos \varphi, \rho \sin \varphi) \in \Gamma_{b_{k}}
\end{array}\right.
$$

Proof. Let $(z, w)=(\rho \cos \varphi, \rho \sin \varphi) \in \Gamma_{a_{k}}$. Consider the solution $(\bar{\rho}(t, \rho, \varphi)$, $\bar{\varphi}(t, \rho, \varphi))$ of (4.6). It follows from (2.3) and the second equation of (4.6) that

$$
\bar{\varphi}^{\prime}(t, \rho, \varphi) \leq-A_{1},
$$

provided that $\rho$ is sufficiently large.

Since the solution $(\bar{z}(t, z, w), \bar{w}(t, z, w))$ of $\left(2.1^{\prime}\right)$ has the least period $\tau\left(a_{k}\right)$, we see that the time in which $\bar{\varphi}(t)$ has a decrement $2 \pi$ is just $\tau\left(a_{k}\right)$. Write

$$
\Phi(\rho, \varphi)=\bar{\varphi}(2 \pi, \rho, \varphi)-\varphi=\bar{\varphi}(2 \pi)-\bar{\varphi}(0)=-2 l \pi-\sigma,
$$

where $l \geq 0$ is an integer, and $0 \leq \sigma<2 \pi$. Let $t_{\sigma}$ denote the time in which $\bar{\varphi}(t)$ decreases from $\varphi-2 l \pi$ to $\varphi-2 l \pi-\sigma$. Then we have

$$
l \cdot \tau\left(a_{k}\right)+t_{\sigma}=2 \pi \text {. }
$$


Since $0 \leq t_{\sigma}<\tau\left(a_{k}\right)$, we obtain

$$
2 \pi=l \cdot \tau\left(a_{k}\right)+t_{\sigma}<(l+1) \tau\left(a_{k}\right) \leq(l+1)\left(\frac{2 \pi}{m}-\alpha\right) .
$$

It follows that $l \geq m$. If $l \geq m+1$, we have

$$
\Phi(\rho, \varphi) \leq-2 l \pi \leq-2(m+1) \pi .
$$

Now, assume $l=m$. Then we have

$$
t_{\sigma}=2 \pi-m \cdot \tau\left(a_{k}\right) \geq 2 \pi-m\left(\frac{2 \pi}{m}-\alpha\right)=m \alpha .
$$

By (4.8) and (4.10), we obtain

$$
-\sigma=\int_{l \cdot \tau\left(a_{k}\right)}^{l \cdot \tau\left(a_{k}\right)+t_{\sigma}} \bar{\varphi}^{\prime}(t, \rho, \varphi) d t \leq-A_{1} t_{\sigma} \leq-m \alpha A_{1} .
$$

Thus we have

$$
\Phi(\rho, \varphi)=-2 l \pi-\sigma \leq-2 m \pi-m \alpha A_{1} .
$$

Combining (4.9) and (4.11) yields the first inequality of (4.7).

The second inequality of (4.7) can be proved in a similar way. The proof of Lemma 4.1 is then completed.

LEMMA 4.2. Let $\Theta(\gamma, \theta)=\bar{\theta}(2 \pi, \gamma, \theta)-\theta$. Then there exists $\gamma_{0}>0$ such that, for $\gamma \geq \gamma_{0}$,

$$
|\Theta(\gamma, \theta)-\Phi(\gamma, \theta)|=|\bar{\theta}(2 \pi, \gamma, \theta)-\bar{\varphi}(2 \pi, \gamma, \theta)|<\beta .
$$

ProOF. Let $(\bar{z}(t, x, y), \bar{w}(t, x, y))$ be the solution of $\left(2.1^{\prime}\right)$ through the initial point $(\bar{z}(0), \bar{w}(0))=(x, y)$. Let

Then we have

$$
\left\{\begin{array}{l}
u(t)=u(t, x, y)=\bar{x}(t, x, y)-\bar{z}(t, x, y) \\
v(t)=v(t, x, y)=\bar{y}(t, x, y)-\bar{w}(t, x, y)
\end{array}\right.
$$

$$
\frac{d u}{d t}=v, \quad \frac{d v}{d t}=p(t)-g^{\prime}(\sigma(t)) u,
$$

where $\sigma(t)=\bar{x}(t)+\lambda(t)(\bar{z}(t)-\bar{x}(t)), 0 \leq \lambda(t) \leq 1$.

Let $\eta(t)=\left(u^{2}(t)+v^{2}(t)\right)^{1 / 2}$. Then we have

$$
\eta \frac{d \eta}{d t}=u v+p(t) v-g^{\prime}(\sigma(t)) u v
$$

It follows from $\left(\mathrm{H}_{1}\right)$ that

$$
\left|\frac{d \eta}{d t}\right| \leq \frac{1}{2}(1+K) \eta+B,
$$

where $B$ is a bound of $|p(t)|$ in $[0,2 \pi]$. The differential inequality (4.13) together with $\eta(0)=0$ yields

$$
\eta(t) \leq \frac{2 B}{K+1}\left[e^{(K+1) \pi}-1\right] \equiv H_{0}
$$

for $t \in[0,2 \pi]$. 
Next write $\psi(t)=\psi(t, \gamma, \theta)=\bar{\theta}(t, \gamma, \theta)-\bar{\varphi}(t, \gamma, \theta)$, where $(\gamma, \theta)$ is the polar coordinate of $(x, y)$, i.e., $(\gamma \cos \theta, \gamma \sin \theta)=(x, y)$. It is clear that if $|\psi(t)|<\pi$, then $\psi(t)$ is just the angle between the vectors $(\bar{x}(t), \bar{y}(t))$ and $(\bar{z}(t), \bar{w}(t))$. By the law of cosines, we have

$$
\cos \psi(t)=\frac{\bar{\gamma}^{2}(t)+\bar{\rho}^{2}(t)-\eta^{2}(t)}{2 \bar{\gamma}(t) \bar{\rho}(t)} \geq 1-\frac{H_{0}^{2}}{2 \bar{\gamma}(t) \bar{\rho}(t)} .
$$

On the other hand, we have $\bar{\gamma}(t) \geq \bar{\rho}(t)-\eta(t) \geq \bar{\rho}(t)-H_{0}$. Therefore, under the assumption that $|\psi(t)|<\pi$ and $\bar{\rho}(t)-H_{0}>0$, we have

$$
\cos \psi(t) \geq 1-\frac{H_{0}^{2}}{2 \bar{\rho}(t)\left(\bar{\rho}(t)-H_{0}\right)} .
$$

Note that

$$
F(\gamma, \varphi)=\inf _{0 \leq t \leq 2 \pi} \bar{\rho}(t)=\inf _{0 \leq t \leq 2 \pi} \bar{\rho}(t, \gamma, \varphi)
$$

becomes arbitrarily large if $\gamma$ is sufficiently large. It follows that there is a constant $\gamma_{0}>0$ such that, for $\gamma \geq \gamma_{0}$ and $t \in[0,2 \pi]$,

$$
\bar{\rho}(t)-H_{0}>0 ; \quad \frac{H_{0}^{2}}{2 \bar{\rho}(t)\left(\bar{\rho}(t)-H_{0}\right)}<1-\cos \delta,
$$

where $\delta=\min (\pi / 2, \beta)$. From (4.15) and (4.16), we conclude that if $|\psi(t)|<\pi$ and $t \in[0,2 \pi]$, then the inequality

$$
|\psi(t)|<\delta
$$

holds.

Since $\psi(0)=0$ and $\psi(t)$ varies continuously as $t$ increases from 0 to $2 \pi$, we can see that (4.17) holds for any $t \in[0,2 \pi]$. In particular, we have

$$
|\psi(2 \pi)|=|\bar{\theta}(2 \pi, \gamma, \theta)-\bar{\varphi}(2 \pi, \gamma, \theta)|<\delta \leq \beta,
$$

for $\gamma \geq \gamma_{0}$. This proves Lemma 4.2.

PROOF OF THEOREM 2.1. Let $c_{1} \geq c_{0}$ be so large that $(\gamma, \theta) \in \Gamma_{c}$ with $c \geq c_{1}$ implies $\gamma \geq \gamma_{0}$, where $\gamma_{0}$ is specified in Lemma 4.2. There is no loss of generality to assume $a_{k} \geq c_{1}$ for $k \geq n_{0}$. It follows that $\bar{\gamma}(t, \gamma, \theta) \geq \bar{\rho}(t)-H_{0}>0, t \in[0,2 \pi]$, provided that $(\gamma, \theta) \in \bar{A}_{k}$ for $k \geq n_{0}$.

Thus the restriction $T \mid A_{k}$ can be written in (4.3), where the function $\bar{\theta}(2 \pi, \gamma, \theta)$ is continuous on $A_{k}$ and satisfies the relation (4.4). Now, we put the integer $l=m$ in (4.3). Then (4.3) can be rewritten in the form of

$$
\gamma^{*}=\bar{\gamma}(2 \pi, \gamma, \theta), \quad \theta^{*}=\theta+\Theta_{1}(\gamma, \theta)
$$

with $\Theta_{1}(\gamma, \theta)=\Theta(\gamma, \theta)+2 m \pi$. By Lemma 4.2, we obtain

$$
\left|\Theta_{1}(\gamma, \theta)-\Phi(\gamma, \theta)-2 m \pi\right|<\beta
$$

which together with (4.7) yields

$$
\begin{array}{ll}
\Theta_{1}(\gamma, \theta)<0, & (\gamma \cos \theta, \gamma \sin \theta) \in \Gamma_{a_{k}} \\
\Theta_{1}(\gamma, \theta)>0, & (\gamma \cos \theta, \gamma \sin \theta) \in \Gamma_{b_{k}},
\end{array}
$$

for $k \geq n_{0}$, where $n_{0}$ is large enough.

This proves the validity of condition (iii) of Theorem $\mathrm{A}$ for the restriction $T \mid \mathcal{A}_{k}$ 
$\left(k \geq n_{0}\right)$. Since $\gamma \geq \gamma_{0}$ implies $\bar{\gamma}(2 \pi, \gamma, \theta) \geq \bar{\rho}(2 \pi)-H_{0}>0$, so the condition (ii) of Theorem A can be easily verified. By Lemma 2.1, the condition (i) of Theorem $A$ also holds. Therefore, we can apply Theorem $A$ to ensure the existence of at least two fixed points of $T$ in $A_{k}\left(k \geq n_{0}\right)$. This means that (1.1) has at least two $2 \pi$-periodic solutions with initial points in $A_{k}$. In the same way, we can prove that $T$ has at least two fixed points in $B_{k}$ which correspond to two $2 \pi$-periodic solutions of (1.1). Since each periodic solution of (1.1) is bounded by a $\Gamma_{6}$, so the above-specified $2 \pi$-periodic solutions of (1.1) constitute an infinite class.

The proof of Theorem 2.1 is thus completed.

5. Finally, we give a concrete example for applications of Theorem 2.1 .

Let $m$ be a positive integer, and let

$$
\bar{g}(x)=x\left[m^{2}+\frac{1}{10} \cos \log \left(1+x^{2}\right)-\frac{x^{2}}{10\left(1+x^{2}\right)} \sin \log \left(1+x^{2}\right)\right] .
$$

A direct calculation yields

$$
m^{2}-\frac{1}{2} \leq \bar{g}^{\prime}(x) \leq m^{2}+\frac{1}{2} .
$$

It follows that $\left(\mathrm{H}_{1}\right)$ and $\left(\mathrm{H}_{2}\right)$ hold for $\bar{g}(x)$.

Consider the auxiliary equation

$$
\frac{d^{2} z}{d t^{2}}+\bar{g}(z)=0 .
$$

According to (2.2), we have

$$
V(z, w) \equiv \frac{1}{2} w^{2}+\bar{G}(z)=c,
$$

where

$$
\bar{G}(z)=\int_{0}^{z} \bar{g}(u) d u=\frac{1}{2} z^{2}\left[m^{2}+\frac{1}{10} \cos \log \left(1+z^{2}\right)\right] .
$$

Take $h=h(c)>0$ such that $\bar{G}(h(c))=c$ : Since $\bar{G}(z)$ is an even function, we have

$$
\tau(c)=2 \sqrt{2} \int_{0}^{h(c)} \frac{d u}{\sqrt{c-\bar{G}(u)}}=\frac{4}{\sqrt{Q(h)}} \int_{0}^{1} \frac{d \xi}{\sqrt{1-\xi^{2} Q(h \xi) / Q(h)}}
$$

where

$$
Q(x)=m^{2}+\frac{1}{10} \cos \log \left(1+x^{2}\right) .
$$

Let $\alpha_{k}=\sqrt{e^{2 k \pi}-1}, \beta_{k}=\sqrt{e^{(2 k+1) \pi}-1}, a_{k}=\bar{G}\left(\alpha_{k}\right)$ and $b_{k}=\bar{G}\left(\beta_{k}\right)$. Then

$$
a_{k}<b_{k} \quad(k=1,2, \ldots)
$$

and

$$
\lim _{k \rightarrow \infty} a_{k}=\lim _{k \rightarrow \infty} b_{k}=\infty .
$$

Since the inequalities

$$
0<\frac{Q\left(\alpha_{k} \xi\right)}{Q\left(\alpha_{k}\right)} \leq 1, \quad \frac{Q\left(\beta_{k} \xi\right)}{Q\left(\beta_{k}\right)} \geq 1
$$


hold for $\xi \in[0,1]$, we have

$$
\begin{aligned}
\tau\left(a_{k}\right) & =\frac{4}{\sqrt{Q\left(\alpha_{k}\right)}} \int_{0}^{1} \frac{d \xi}{\sqrt{1-\xi^{2} Q\left(\alpha_{k} \xi\right) / Q\left(\alpha_{k}\right)}} \\
& \leq \frac{4 \sqrt{10}}{\sqrt{10 m^{2}+1}} \int_{0}^{1} \frac{d \xi}{\sqrt{1-\xi^{2}}} \leq \frac{2 \pi}{m}-\bar{\alpha}
\end{aligned}
$$

and

where

$$
\begin{aligned}
\tau\left(b_{k}\right) & =\frac{4}{\sqrt{Q\left(\beta_{k}\right)}} \int_{0}^{1} \frac{d \xi}{\sqrt{1-\xi^{2} Q\left(\beta_{k} \xi\right) / Q\left(\beta_{k}\right)}} \\
& \geq \frac{4 \sqrt{10}}{\sqrt{10 m^{2}-1}} \int_{0}^{1} \frac{d \xi}{\sqrt{1-\xi^{2}}} \geq \frac{2 \pi}{m}+\bar{\alpha}
\end{aligned}
$$

$$
\bar{\alpha}=\frac{2 \pi}{m} \cdot \min \left\{1-\frac{\sqrt{10} m}{\sqrt{10 m^{2}+1}}, \frac{\sqrt{10} m}{\sqrt{10 m^{2}-1}}-1\right\} .
$$

This proves that $\left(\mathrm{H}_{3}\right)$ is also valid for $\bar{g}(x)$.

Therefore, the function $\bar{g}(x)$ satisfies all the assumptions of Theorem 2.1, and the corresponding equation

$$
\frac{c^{2} z}{d t^{2}}+\bar{g}(x)=p(t)
$$

has infinitely many $2 \pi$-periodic solutions for any $2 \pi$-periodic function $p(t) \in C(R, R)$.

The author is grateful to $W$. Y. Ding for many valuable suggestions. The author also extends his thanks to Dr. George R. Sell for his invaluable assistance.

\section{REFERENCES}

1. W. Y. Ding, Fixed points of twist mappings and periodic solutions of ordinary differential equations, Acta Math. Sinica 25 (1981), 227-235.

2. D. E. Leach, On Poincare's perturbation theorem and a theorem of W. S. Loud, J. Differential Equations 7 (1970), 34-53.

3. R. Reissig, Contractive mappings and periodically perturbed, non-conservative systems, Atti Accad. Naz. Lincei Rend. Cl. Sci. Fis. Mat. Natur. 58 (1975), 696-702.

4. A. C. Laser and D. E. Leach, Bounded perturbations of forced harmonic oscillations at resonance, Ann. Mat. Pura Appl. 82 (1969), 49-68.

5. L. Césari, Nonlinear problems across a point of resonance for non-self-adjoint systems, non-linear analysis (A Collection of Papers in Honor of Erich H. Rothe), edited by L. Césari et al., Academic Press, New York, 1978, pp. 43-67.

6. T. R. Ding, Nonlinear oscillations at a point of resonance, Sci. Sinica (Chinese), Series A, 1 (1982), 1-13.

7. W. Y. Ding, A Generalization of the Poincare-Birkhoff Theorem, Proc. Amer. Math. Soc. (to appear).

Department of Mathematics, Peking University, Bei-Jing, The People's REPUBLIC OF CHINA 